\title{
Pericyte Polarity is Dependent on Location Along the Retinal Vasculature
}

\author{
Lauren Azrin
}

White Plains High School, 550 North Street, White Plains, NY, 10605, USA; Laa7green@gmail.com

\begin{abstract}
Pericytes are contractile cells that surround the microvasculature, highly concentrated in the retina. As blood flows from arteries to veins through capillaries, the pericyte structure allows them to control blood flow. However, it is not known if these cells have spatial differences in shape and structure (polarity) that would enable them to carry out specialized functions. The goal of this research was to understand how pericytes control blood flow by examining their structural symmetry along different parts of the microvasculature, and to see if structure is consistent with the direction of blood flow. Through dissection and imaging of mouse retinas, it was found that while the smooth muscle cells in large vessels are overall symmetrical, pericytes are polar and asymmetrical, with the artery side significantly longer and wider than the vein side. This suggests that pericytes help circulate blood by using the longer and wider artery side to communicate, control, and coordinate the blood flow. This helps to understand the movement of blood in response to a stimulus in various disciplines, such as imaging, or to better comprehend certain diseases, such as diabetic retinopathy, characterized by a decline in pericyte fnction or number.

KEYWORDS: Eyes; Cell structure; Vasculature; Retina; Pericytes.
\end{abstract}

\section{Introduction}

Pericytes, also referred to as a "forgotten cell type,"1 are multi-functional cells embedded in the walls of capillaries (Figure 1) throughout the body, including the brain and the retina where they are highly concentrated. Little attention has been paid to them during the previous century, but they have recently been identified for regulating cerebral and retinal blood flow, maintaining the blood-brain barrier (BBB) and the blood-retinal barrier (BRB), and controlling vascular development and angiogenesis.

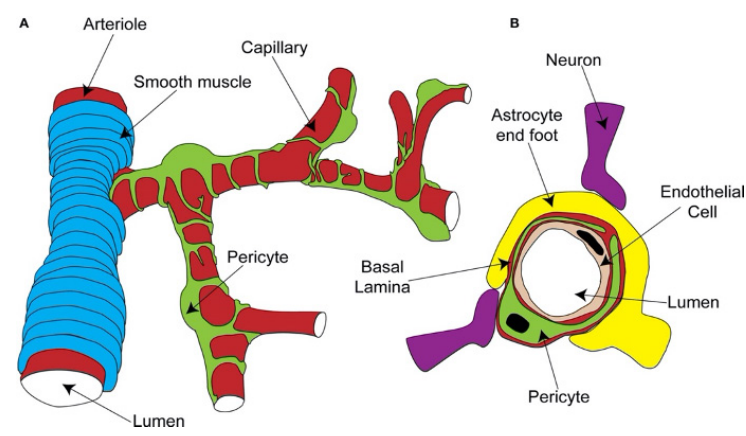

Figure 1: What a pericyte looks like as it wraps around the capillary. ${ }^{2}$

Until recently, it has been difficult to accurately characterize the sub-types of pericytes, or to specifically target them to alter shape or function. New techniques are beginning to be developed both in vitro and in vivo that will improve the investigation of pericytes and help to identify their possible roles in diseases. Pericyte dysfunction is growing in recognition as a contributor to the development of vascular diseases like diabetic retinopathy - associated with strokes - and neurodegenerative diseases like Alzheimer's disease. ${ }^{3}$ Pericyte role and function has been largely neglected in past research. Besides knowledge of their location, wrapped along blood microvessels, little is known about how they interact with these vessels or their exact shape.

It has been shown that pericyte dropout or loss may have consequences on the eyes of diabetics affected by diabetic retinopathy, a disease that occurs with an excess buildup of sugar. ${ }^{4}$ This leads to abnormalities that can be clinically observed. Pericyte loss has been defined as characteristic of the diabetic retina; therefore, retinal capillary coverage with pericytes is crucial for the survival of endothelial cells, especially under stress conditions like diabetes. ${ }^{4,5}$ More exploration into pericyte shape and function could help to clarify the mechanisms of pericyte loss in order to develop new effective drugs for the prevention of diabetic retinopathy.

A more recent study tried to connect pericyte function with blood flow since they surround blood vessels in the retina and found that the density of pericytes and their reactivity to stimulation were reduced in diabetes. ${ }^{6}$ The study focused on the regulation of capillary diameter by pericytes, showing how disruption of this balance can contribute to a decline in control in diabetes. Another recent study revealed that pericytes play a key role in the regulation of the vasculature and blood flow. ${ }^{7}$ Because diabetic retinopathy can develop due to blood flow complications, a loss or decline in function of pericytes could have a similar role and help to understand this relation. Therefore, using a mouse model of diabetic retinopathy to get a closer look into pericyte shape and location and a clearer picture of how they contribute to this phenomenon could be of help.

It is important to take images of pericytes in different locations along the retinal microvasculature to get a better understanding of where they are located in relation to the major vessels. By dissecting and fixing retinal tissue using paraformaldehyde fixation and methods described by Ivanova et al., vessels in the tissue can be imaged under a confocal microscope. ${ }^{8}$ Ret 
inal whole-mount observation by confocal laser technology is the only method that allows a three-dimensional analysis of the entire retina. ${ }^{9}$ Mapping pericytes helps to get a full picture of their relative location and if this has a role in their function. Moreover, measuring both sides of the pericyte, along with its area, can help researchers determine if pericyte arms favor one side in particular and if this may have an effect on pericyte function and/or control on blood flow from the artery to the vein. Putting pericyte's shape in perspective with the direction of blood flow will help to understand their movement and constriction.

The shape of pericytes, and their polarity, as well as mapping them out along the layers of vessels in the retina to look for any trends, has not been addressed in previous literature. This study will include images of numerous pericytes in different layers and use precise measurements to analyze and classify them. Pericytes contain contractile proteins that may enable them to initiate signaling, suggesting that blood flow control originates in the retinal microvessels where pericytes monitor capillary diameter, also in response to changes in neural activity, which may in turn contribute to vascular disease. ${ }^{10}$ Pericytes contract the blood vessels they surround and have an effect on the amount of blood flow, with arms extending from the feeding arteries to the finest capillaries, and it is previously known that blood flows in one way from arteries to veins (Figure 2). ${ }^{11}$
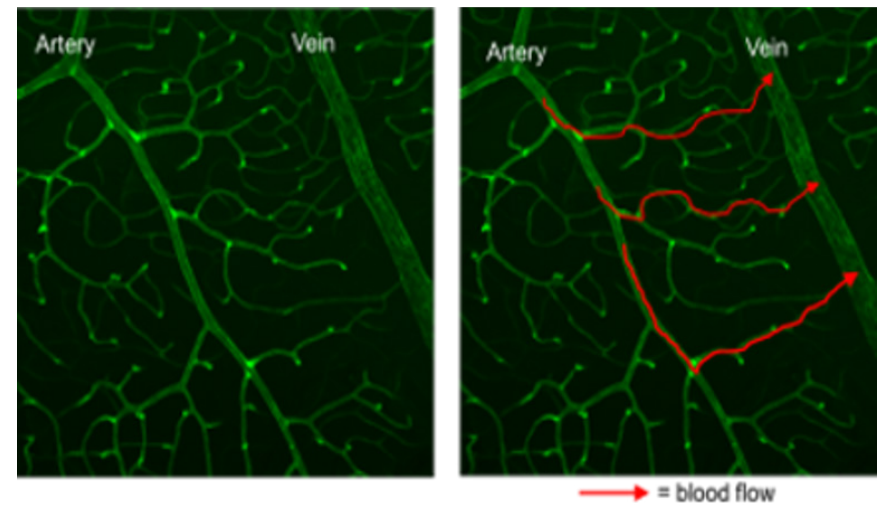

Figure 2. (a): An image of the retinal blood vessels taken by a confocal microscope. (b): The direction that blood flows from artery to vein within the vascular system.

\section{Statement of Purpose:}

The hypothesis of this paper is that the pericytes will be longer and have a greater area on the artery facing side because of their prominence in the movement of blood flow and their role in communication during a stimulus.

\section{Methods}

First, the eyes of two female mice (4 eyes) were obtained. The eyes had a GCaMP6f calcium sensor under the NG2 promoter; pericytes in the retina are positive for NG2, so this sensor made them fluorescent and able to be mapped out through the different retinal layers. The other two were a result of a crossbreed in the lab of a leaky cyclization recombination (Cre) mouse that resulted in the individual staining of the pericytes, which is rare since they are usually interconnected. These were specifically targeted and able to be measured.
The retinas were then placed in hydroxyethyl piperazineethhanesulfonic acid (HEPES) solution to be dissected by a process outlined by Ivanova et al. ${ }^{8}$ While in the HEPES solution, the cornea was first cut in a circular direction to be removed. Then the lens was carefully removed. At this stage in the dissection the eye cap with the retina still attached was fixed in a solution of paraformaldehyde (PFA). After fixing for 15 minutes on an oscillator, the PFA was removed, and the eye cap and retina were washed twice in a phosphate-buffered saline solution (PBS) while remaining on the oscillator. After washing, another thin layer of the eye cap as well as the optic nerve were cut to slowly undo attachments to the eye cap and free the retina. Retinas were then transferred into individual wells with $1 \mathrm{~mL}$ PBS solution and kept in humidified atmosphere to prevent them from drying. Any retina that had folded over in the process was carefully unfolded. Then, they were cut in 4 places so that they could be flattened for whole mounting. Once the retinas were flattened, the PBS was removed and $0.5 \mathrm{~mL}$ of chemiblock triton sodium azide (CTA), a permeabilizing solution, was added and changed every hour to maintain hydration. The retinas were kept in CTA at $4^{\circ} \mathrm{C}$ overnight in enough solution to keep them from dying out, but not enough to cause flotation and folding.

Then, the retinas were stained red with isolectin Alexa 568. Isolectin sticks to the blood vessels and Alexa 568 stains isolectin with a fluorescent label, making the vessels appear red under a microscope. The vessels were stained overnight and then washed with $1 \mathrm{~mL}$ of PBS two times to make sure the retinas were fully clean. The wells were then put back on the oscillator, covered in tinfoil to protect the now fluorescent retinas from the light. The retinas were then whole mounted for imaging under the confocal microscope.

Images were taken of numerous pericytes individually found throughout different layers of the vasculature. Each pericyte location along the vascular tree was analyzed to determine which side was facing the artery and which side was facing the vein. Every pericyte imaged was then classified by which point in the vasculature it was in and mapped out on the whole retina to determine a relation between location and structure.

After imaging under the microscope, each pericyte observed was measured for its length in micrometers and area (pixel count) on both the artery and vein facing side, not including the soma, using ImageJ software (Figure 3). A total of 40 pericytes from both eyes were collected and analyzed. The 2 eyes of the second mouse were used to locate pericytes along the retinal vasculature and analyze shape. The statistical analyses were performed on Microsoft Excel using homoscedastic one-tailed t-tests between the two average areas (artery facing side and vein facing side) and the two average lengths (artery facing side and vein facing side).

\section{Results and Discussion}

First, our results show that pericytes are polar, or asymmetrical in arm length, and do not all have one universal shape or symmetry, like many other types of cells do. In images of the vessels, the pericytes were not symmetrical on the sides facing 
the vein compared to the artery, suggesting polarity. The sides to the left and right of the soma (head of the cell or cell body) that branch into other directions, varied in length, width, and shape as they wrapped around the vessel (Figure 3).

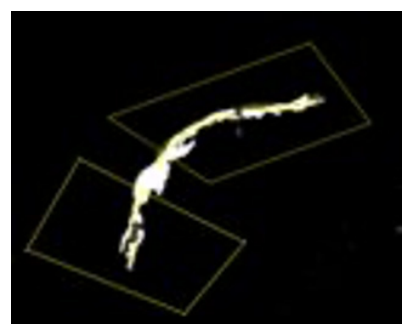

Figure 3: Displays how each pericyte was thresholded and zoomed in on through the ImageJ software to effectively measure the length and the pixel count of both the artery and vein side. This is a flattened image of how one specific pericyte looked like when being measured on ImageJ software.

It was also found that the smooth muscle cells located on the main large vessels in the superficial layer are overall symmetrical. All the smooth muscle cells located on the major arteries that branch out directly from the center of the retina in the superficial layer were symmetrical and did not express polarity; both sides appeared similar in structure (Figure 4).

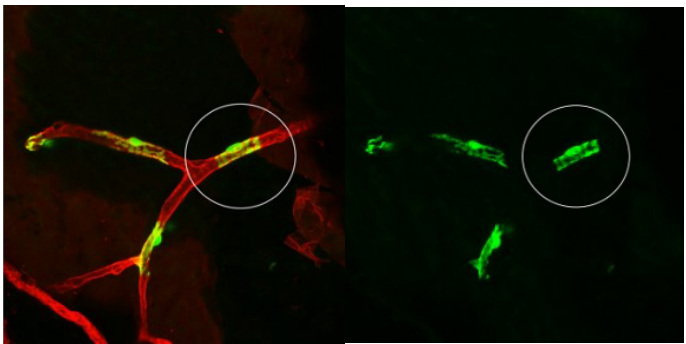

Figure 4: The smooth muscle cells located on major arteries, displaying a symmetrical structure. The left image shows the cells only, and the right image shows the same cells located on the vessels. This is achieved on ImageJ software by inactivating the channel that displays the vessels to get a closer look at the pericytes.

We also found that the hierarchy within the vascular tree, meaning the layer of the retina that pericytes are in, dictates their shape (Figure 5).

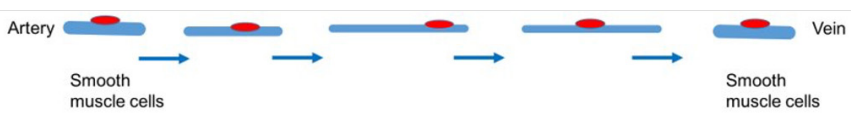

Figure 5: An image displaying the changes in pericyte structure depending on their relative closeness to arteries or veins. The blue represents the arms of the cell and the red represents the soma or cell body.

Moreover, it was found when comparing vein length and artery length that the artery side is longer than the vein side (Figure 6), and this did prove significant with statistical analyses (Figure 7).

Lastly, it was found that when comparing the vein area and the artery area, that the artery side has a larger area (pixel count) than the vein side (Figure 8), and this did prove significant with statistical analyses (Figure 9).

\section{- Conclusions}

This study helps to understand the structure and function of the pericyte in relation to blood flow, which has not been largely covered in past research. By determining their structu ral significance in favor of the artery, being longer and wider on its related side, it can be concluded that pericytes help to circulate blood by using the longer and wider (indicating more muscular) side to communicate, control, and coordinate blood circulation in the vascular tree.
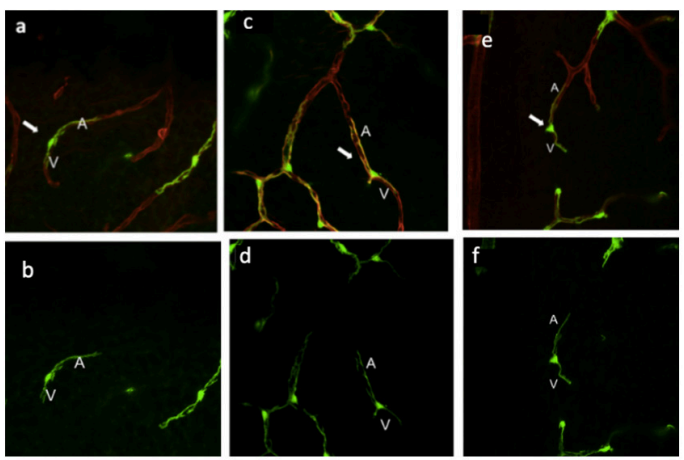

Figure 6: Representative images of pericytes, with the arrow identifying the pericyte being measured. $A=$ artery facing side, $V=$ vein facing side. It is clear that the artery sides are longer than the vein sides. Again, each pericyte is shown with and without the vessels.

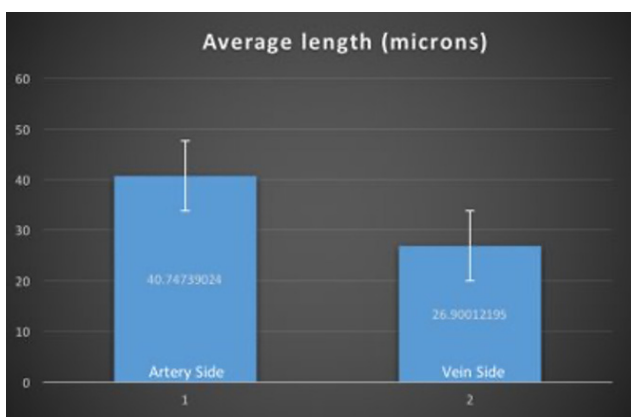

Figure 7: The bar graph of the average length in microns of the artery and vein facing side of all 40 pericytes. When a one-tailed t-test was applied, the p-value was less than 0.001 , demonstrating that the artery facing side was significantly longer.

This information can be used to understand the movement of blood in response to a stimulus in various disciplines, such as in imaging (the BOLD contrast MRI or optogenetics) or targeting certain diseases that occur when there is a decline in pericyte function or number, like diabetic retinopathy, neurovascular diseases, and neurological diseases. This information could also help to explain pericyte structure and role in blood circulation, so that if they were to be impaired during certain diseases, there is a baseline for what they should look like and how they should function in relation to blood flow.
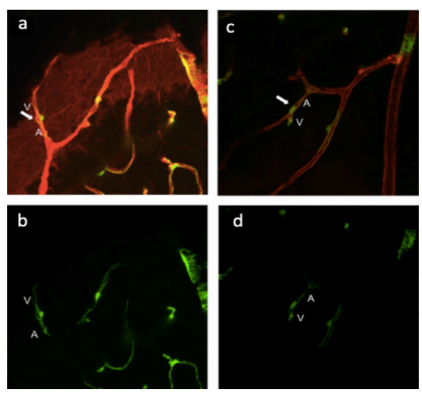

Figure 8: Representative images of pericytes, with the arrow pointing to the pericyte being measured and the $\mathrm{A}$ and $\mathrm{V}$ standing for artery facing side and vein facing side. Again, each pericyte is shown with and without the vessels. The artery facing side appears to have a larger area, and this proved true by pixel counting through ImageJ software. 


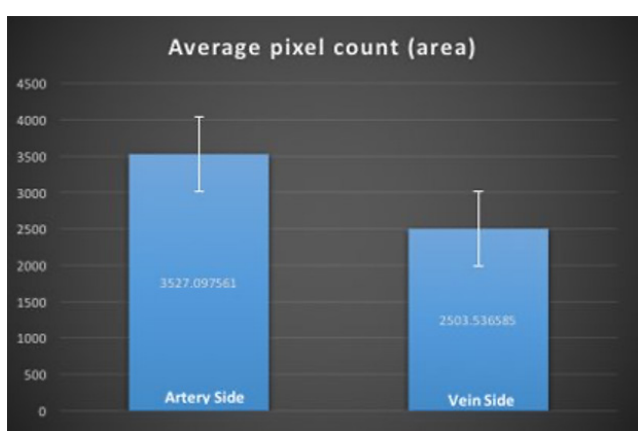

Figure 9: The bar graph of the average area or pixel count of the artery and vein facing side of all 40 pericytes. When a one-tailed t-test was applied, the $\mathrm{p}$-value was less than 0.05 , demonstrating that the artery facing side had a significantly larger area.

Future studies can look at pericytes under a 2-photon microscope to assess not just fixed retinas and pericytes, but live ones to see how pericyte shape changes as blood flows and to get an even further understanding of how they contribute to movement in the vascular tree.

\section{Acknowledgements}

I would like to thank my mentors at Burke Neurological Institute: Botir Sagdullaev, Ph.D., Elena Ivanova, Ph.D., and Paola Bianchimano, Ph.D. I would also like to thank my science research teacher, Ms. Fleming, for all her continued support and guidance.

\section{- References}

1. Bergers, G. and Song, S. (2005). The role of pericytes in bloodvessel formation and maintenance. Neuro-Oncology, 7(4), pp.452464.

2. Hamilton, N. (2010). Pericyte-mediated regulation of capillary diameter: a component of neurovascular coupling in health and disease. Frontiers in Neurogenetics, 2.

3. Brown, L., Foster, C., Courtney, J., King, N., Howells, D. and Sutherland, B. (2019). Pericytes and Neurovascular Function in the Healthy and Diseased Brain. Frontiers in Cellular Neuroscience, 13.

4. Beltramo, E. and Porta, M. (2013). Pericyte Loss in Diabetic Retinopathy: Mechanisms and Consequences. Current Medicinal Chemistry, 20(26), pp.3218-3225..

5. Hammes, H. (2005). Pericytes and the Pathogenesis of Diabetic Retinopathy. Hormone and Metabolic Research, 37, pp.39-43.

6. Ivanova, E., Kovacs-Oller, T. and Sagdullaev, B. (2017). Vascular Pericyte Impairment and Connexin43 Gap Junction Deficit Contribute to Vasomotor Decline in Diabetic Retinopathy. The Journal of Neuroscience, 37(32), pp.7580-7594.

7. Santos, G., Prazeres, P., Mintz, A. and Birbrair, A. (2017). Role of pericytes in the retina. Eye, 32(3), pp.483-486.

8. Ivanova, E., Toychiev, A., Yee, C. and Sagdullaev, B. (2013). Optimized Protocol for Retinal Wholemount Preparation for Imaging and Immunohistochemistry. Journal of Visualized Experiments, (82).

9. Ramos, D. (2013). The Use of Confocal laser Microscopy to Ana lyze Mouse Retinal Blood Vessels. IntechOpen.

10.Peppiatt, C., Howarth, C., Mobbs, P. and Attwell, D. (2006). Bidirectional control of CNS capillary diameter by pericytes. Nature, 443(7112), pp.700-704.

11.Ivanova, E., Kovacs-Oller, T. and Sagdullaev, B. (2019). Domainspecific distribution of gap junctions defines cellular coupling to establish a vascular relay in the retina. Journal of retina. Journal of Comparative Neurology.

\section{Author}

Lauren Azrin is a Senior at White Plains High School in White Plains, NY. She enjoys studying biology and is interested in continuing her ophthalmology research in college. 